\title{
Avaliação da mudança de currículo no curso de ciência econômicas da universidade federal de Santa Catarina em 2009
}

Resumo: A mudança do currículo do curso de Ciências Econômicas da UFSC em 2009 foi proposta com o intuito de flexibilizar o curso, e formar economistas mais bem preparados para o mercado de trabalho. Este artigo apresenta um exercício de avaliação da mudança sobre indicadores dos resultados acadêmicos dos alunos: reprovação, abandono e nota padronizada. A amostra analisada foi definida como estudantes da quarta fase do curso de Ciências Econômicas matriculados em 2007 como grupo de controle e 2010 como grupo de tratamento. Encontram-se indicativos que a mudança no currículo aumentou a probabilidade de abandono, aumentou a probabilidade de reprovação e o número de reprovações, e diminuiu a nota padronizada dos alunos.

Palavras chaves: Ciências Econômicas; Mudança de Currículo; Avaliação de Impacto

\begin{abstract}
The change in the curriculum of the Economic Sciences course at UFSC in 2009 was proposed in order to make the course more flexible and to train economists better prepared for the market. This article presents an evaluation exercise of the change in indicators of the academic results of the students: failure, abandonment and standardized mark. The analyzed sample was defined as students of the fourth phase of the Economic Sciences course enrolled in 2007 as a control group and 2010 as a treatment group. It is indicative that the change in the curriculum increased the probability of dropout, increased the probability of disapproval and the number of disapprovals, and decreased the students' standardized grade
\end{abstract}

Keywords: Economics Science. Curriculum Change. Impact Evaluation.

JEL: A21, C35. 


\section{INTRODUÇÃ̃}

A história do curso de Ciências Econômicas da Universidade Federal de Santa Catarina (UFSC) teve seu início no curso de Administração e Negócios na Academia do Comércio, com a primeira aula acontecendo em 01/03/1943, sendo o segundo mais antigo curso de nível superior funcionando ininterruptamente em Santa Catarina. Sua grade curricular incluía disciplinas como Economia Política, Matemática Financeira, Contabilidade Pública, Finanças e Economia Bancária, etc. No entanto, o documento que oficializa a Faculdade Ciências Econômicas data de 22/05/1954 (VIEIRA; FÉLIX, 2008, p. 85). Em 1955, o curso foi oficialmente reconhecido pelo Ministério da Educação ${ }^{1}$. Atualmente, são ofertadas 180 vagas ao ano, sendo 90 para cada semestre letivo, distribuídas igualmente em dois turnos, diurno e noturno, i.e., 45 vagas para cada turno.

Ao longo da história do curso, aconteceram três mudanças curriculares: em 1986, ocorreu a primeira. A segunda mudança ocorreu em 1995, e em 2009, objeto de estudo deste artigo, ocorreu a terceira. Esta última mudança começou a ser discutida em 2005, quando foi formada a primeira comissão, da qual faziam parte professores, estudantes e profissionais da economia, e que promoveu fóruns de debate, até que uma proposta foi elaborada pra novas discussões.

Em 2008, uma nova comissão foi formada, de maneira a sistematizar a proposta de 2005, que culminaria com a reforma curricular, através da submissão, e posterior aprovação, pelo Colegiado do Curso, do Projeto Político Pedagógico (PPP) em junho de 2009, conforme a linha do tempo na figura 1 :

Figura 1. Linha do tempo do Curso de Ciências Econômicas da UFSC

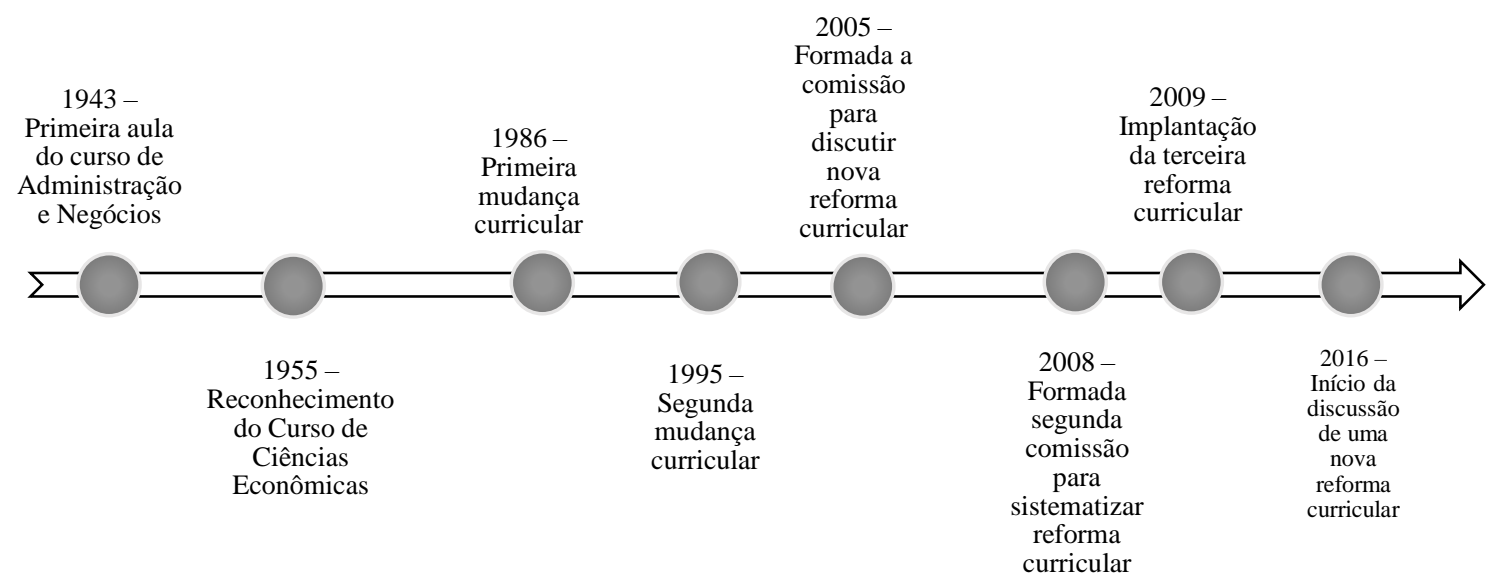

Fonte: elaborada pelo autor (2016)

\footnotetext{
${ }^{1}$ Reconhecido pelo Decreto no 37.994 de 28 de setembro de 1954. Ver (VIEIRA; FÉLIX, 2008)
} 
Na tentativa de atualizar o currículo, cuja última alteração havia ocorrido em 1995 , tentou-se a formação de um consenso baseando-se nas diretrizes curriculares nacionais e com uma preocupação de capacitar e habilitar profissionais competentes e conscientes das necessidades sociais. Assim, o PPP teve como princípios fundamentais a pluralidade metodológica e paradigmática, criação de competência para análise econômica, intensificação da formação teórico-quantitativo nas primeiras fases do curso, etc.

Não obstante, ao conversar com professores da época, verificou-se que o principal objetivo da alteração curricular era a flexibilização do currículo do curso, o que se pressupunha que levaria a um maior interesse dos discentes em permanecer no curso, visto que poderia escolher, livremente, a direção em que seguiria, com a ampliação da oferta de disciplinas optativas. Essa percepção decorria da taxa de abandono, historicamente alta, conforme demonstra um relatório elaborado pelo Núcleo Docente Estruturante (NDE):

Destaque-se que, em números absolutos, o curso de Economia possui o maior número de abandonos de toda a UFSC: são, em média, 102 abandonos a cada ano, perfazendo um padrão contínuo ao longo da última década. Ou seja, mais da metade dos que ingressam em Ciências Econômicas desistem e evadem ao longo do curso (NDE, 2011, p. 11).

Nesse contexto, de forma inédita, o artigo - um exercício para avaliação na disciplina de Avaliação Econômica de Políticas Públicas - busca contribuir para análise da mudança do currículo em 2009, visto que, desde sua implementação, não houve uma análise quantitativa/qualitativa dos efeitos da mudança, e se estes efeitos estão de acordo com o desejado pela comunidade do curso.

Assim, utilizando os dados disponíveis no sistema de administração de dados utilizado pela UFSC, o Sistema de Controle Acadêmico da Graduação (CAGR), buscou-se estimar os efeitos causais, dada uma série de características, em quatro indicadores de impacto do "tratamento", i.e., a mudança no currículo. Isso se justifica principalmente neste atual contexto de discussão de uma nova mudança de currículo, em que discentes e docentes entendem a necessidade de mudanças na direção de melhoria do currículo, visando uma formação mais específica e direcionada para os estudantes.

Os resultados observados dizem respeito a comparação de duas coortes, o que não implica que continuariam os mesmos entre outras coortes. Todavia, se forem comparadas 
coortes mais próximas, perde-se graus de liberdade, enquanto que se forem comparadas coortes mais distantes, aumentam-se os confounders ${ }^{2}$.

Além desta introdução, o artigo conta com mais cinco seções: a segunda seção, que apresenta os indicadores de impacto utilizados, bem como a amostra coletada, a terceira uma revisão da literatura a respeito dos métodos utilizados; a quarta seção apresenta as covariadas, a quinta seção apresenta os resultados, e por fim, a sexta seção apresenta as conclusões e limitações deste artigo, bem como sugestões para pesquisas futuras.

\section{AMOSTRA COLETADA E INDICADORES DE IMPACTO}

As coortes - termo utilizado em estatística para designar um conjunto de pessoas que tem em comum um conjunto de características, ou então um evento que se deu no mesmo período de tempo - foram separadas em duas: a primeira coorte é composta por alunos matriculados antes da mudança do currículo de 2009 e a segunda coorte composta por alunos matriculados depois da mudança do currículo de 2009, com uma diferença no tempo, entre elas, suficientemente pequena (apenas três anos) para não haver diferenças significativas nas características dos alunos, mas suficientemente grande para se obter uma amostra de tamanho razoável para se estimar corretamente os parâmetros. Assim, a única grande diferença que haveria entre estes alunos seria o currículo sob o qual cada um está matriculado. Nesse sentido, o presente artigo se propõe a fazer uma análise comparando as duas coortes, para verificar o efeito do tratamento, i.e., mudança do currículo, no desempenho dos alunos.

A amostra foi definida, então, como alunos que ingressaram no curso no ano de 2007, no primeiro ou segundo semestre, para ser o grupo de controle, independentemente da forma de acesso (vestibular, retorno, transferência, etc.). Esse grupo potencialmente estará na $4^{\text {a }}$ fase do curso no segundo semestre de 2008 , ou no primeiro semestre de 2009 , logo antes da mudança do currículo.

O grupo de tratamento foi definido como alunos que ingressaram no curso, também independentemente da forma de ingresso, no ano de 2010, logo após a mudança do currículo, de maneira que estes alunos potencialmente estariam na $4^{\text {a }}$ fase no segundo semestre de 2011 ou primeiro semestre de 2012.

Para estimarmos o impacto do efeito tratamento, a mudança do currículo, foram escolhidos os seguintes indicadores de impacto:

\footnotetext{
${ }^{2}$ Não há tradução para essa palavra na língua portuguesa. A tradução literal é confundidor (tradução nossa).
} 
- $\quad Y_{i}$, sendo uma dummy caso o aluno tenha deixado o curso até a $3^{\text {a }}$ fase;

- $Y_{i}$, sendo uma dummy caso o aluno esteja cursando a grade sem reprovações até a $4^{\mathrm{a}}$ fase, sob o seu currículo;

- $Y_{i}=0,1,2, \ldots, n$, indicando quantas disciplinas o aluno reprovou até a $4^{\text {a }}$ fase;

- $Y_{i}$, sendo a nota padronizada do aluno, isto é, o Índice de Aproveitamento Acumulado (IAA).

A partir destas hipóteses, roda-se uma regressão para cada $Y_{i}$.

$$
Y_{i}=\beta_{0}+\beta_{1} T_{i}+\sum_{k=2}^{K} \beta_{k} X_{i k}+u_{i}
$$

Sendo $\sum_{k=2}^{K} \beta_{k} X_{i k}$ para cada $X_{i}$ definido como covariadas, para verificar qual foi o impacto que a mudança de currículo causou nas notas dos alunos, se o aluno teve alguma reprovação ou não até a $4^{a}$ fase do curso; se houve algum impacto na desistência ou abandono do curso até a terceira fase; e se houve um aumento no número de reprovações.

Os dois grupos totalizam 252 observações para o indicador de abandono e 171 observações para os indicadores de reprovação, o número de reprovações e o IAA, já que os três últimos indicadores só foram considerados para os estudantes que estavam matriculados regularmente na quarta fase.

\section{REVISÃO DA LITERATURA METODOLÓGICA}

Os dois primeiros indicadores de impacto são variáveis qualitativas, e assumem, assim, valores entre 0 ou 1 . Os modelos de probabilidade linear assumem que a probabilidade de resposta é linear em um conjunto de parâmetros (WOOLDRIDGE, 2007). Para evitar suas limitações, considere uma classe de modelos de resposta binária na seguinte forma:

$$
P(Y=1 \mid x)=F\left(\beta_{0}+\beta_{1} x_{1}+\ldots+\beta_{k} x_{i k}\right)
$$

De tal maneira que 


$$
F\left(\beta_{0}+x_{i} \beta\right)
$$

Em que F é uma função assumindo valores estritamente entre 0 e 1, isto é, $0<\mathrm{F}(\mathrm{z})<1$, para todos os números $z$ reais, garantindo que as probabilidades estimadas de resposta estejam estritamente entre zero e um; e que $x_{i} \beta$ seja um vetor de parâmetros.

A função do modelo logit é:

$$
P_{R}\left(Y_{i} \mid X_{i}\right)=\left[\frac{E X P\left(x_{i} \beta\right)}{1+E X P\left(x_{i} \beta\right)}\right]^{T_{i}} \times\left[\frac{1}{1+E X P\left(x_{i} \beta\right)}\right]^{1-T_{i}}
$$

Assim, a regressão logística terá, como resultado, a probabilidade de não haver abandono, na média, caso o aluno esteja cursando o currículo novo, controlado pelas covariadas., assim como trará o resultado para o segundo indicador escolhido, isto é, a probabilidade de reprovações, dada a mudança do currículo as variáveis de controle.

Dada a não linearidade do modelo, a estimação por Mínimos Quadrados Ordinários não é aplicável. O mais adequado, então, é utilizar a estimação de máxima-verossimilhança, baseada na distribuição de $y$ dado $x$. Assim, a heterocedasticidade na variância de $y$ dado $x$ é automaticamente considerada.

O terceiro indicador de impacto, o número de reprovações, é uma variável de contagem, que assume apenas valores não negativos, inclusive zero, tal que $Y_{i}=0,1,2, \ldots, n$. Observando o seu histograma na figura 1, é possível concluir ainda que a variável respeita uma distribuição de probabilidade de Poisson:

Figura 1. Distribuição de probabilidade da variável Número de reprovações.

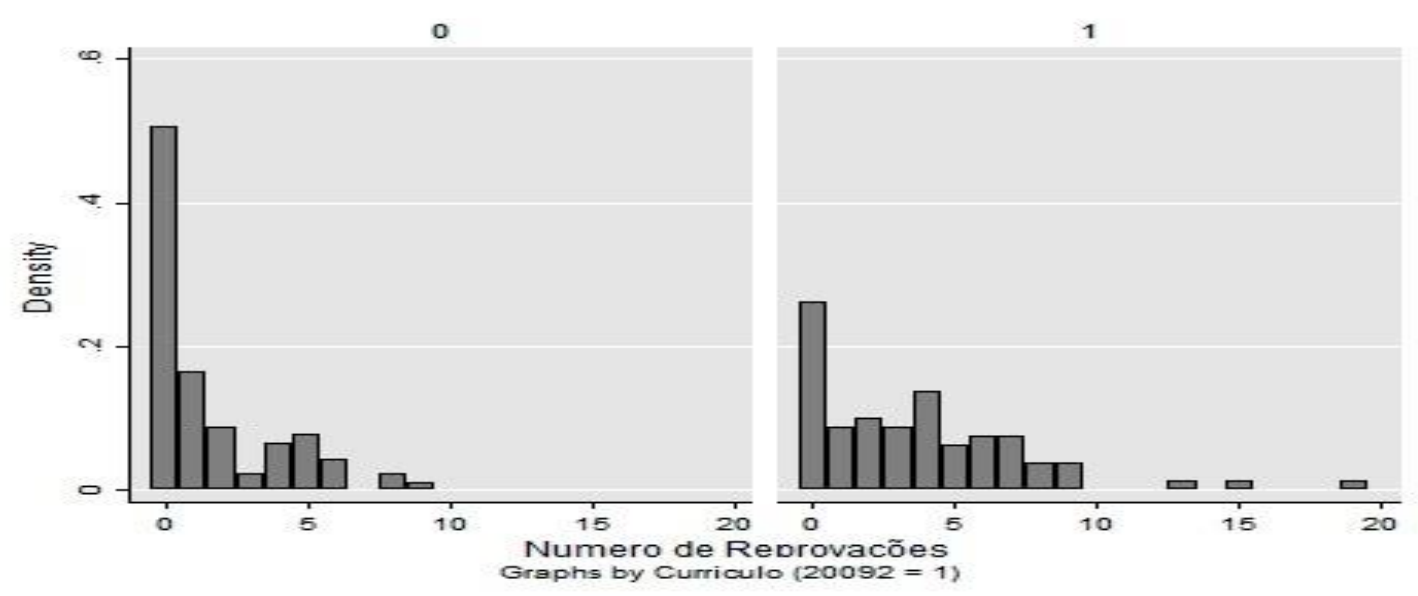

Fonte: CAGR, elaborada pelo autor (2016). 
Pelas mesmas razões discutidas para as variáveis de resposta binárias, um modelo linear para $E\left(Y_{i} \mid x_{1}, x_{2}, \ldots x_{n}\right)$ pode não fornecer o melhor ajuste para todas as variáveis. Ainda, dado que a variável pode assumir o valor de zero, não podemos tomar o seu logaritmo (WOOLDRIDGE, 2012, p. 604).

Assim, o método mais adequado, isto é, o mais eficaz, é modelar o valor esperado $E\left(Y_{i} \mid x_{1}, x_{2}, \ldots x_{n}\right)$ como uma função exponencial, tal que

$$
E\left(Y_{i} \mid x_{1}, x_{2}, \ldots x_{n}\right)=\operatorname{EXP}\left(\beta_{0}+\beta_{1} x_{1}+\ldots+\beta_{k} x_{k}\right)
$$

Como a $\operatorname{EXP}(\cdot)$ é sempre positivo, isso garante que os valores previstos para $Y_{i}$ sejam sempre positivos. A interpretação dos parâmetros deve ser como se tivéssemos uma função linear, com $\log y_{i}$ como variável dependente ${ }^{3}$.

Dado que a equação (4) é não linear em seus parâmetros, não é possível utilizar um modelo de regressão linear para estimação. Considerando que todas as distribuições de dados de contagem exibem heterocedasticidade, o método adequado é o de máxima verossimilhança, utilizado neste artigo.

A função log-verossimilhança pode ser construída, então, da seguinte forma, tornando-a fácil de maximizar, para obter os estimadores:

$$
\sum_{i=1}^{n} \ell_{i}(\beta)=\sum_{i=1}^{n}\left\{y_{i} x_{i} \beta-\exp \left(x_{i} \beta\right)\right\}
$$

Com os parâmetros estimados, é o $\beta_{1}$, da equação 1 que indicará a probabilidade do número de reprovações de um aluno, na média, até a quarta fase, sob o novo currículo.

Por fim, o último indicador é o Índice de Aproveitamento Acumulado (IAA). O IAA é calculado pela razão entre o somatório do produto das notas pela carga horária em cada disciplina (CHC) pela carga horária total cursada nos semestres (CHT). É dado pela seguinte equação:

$$
I A A=\frac{\sum \text { notas } \times C H C}{C H T}
$$

\footnotetext{
${ }^{3}$ Para mais detalhes sobre a função exponencial e a interpretação dos parâmetros, ver (WOOLDRIDGE, 2012)
} 
Dado que o IAA é uma variável linear e discreta, o método adequado para testar a influência da mudança no currículo é uma estimar uma regressão por Mínimos Quadrados Ordinários, para obter um teste de diferença de médias, em que o $\beta_{1}$ estimado indica a diferença do IAA para os alunos matriculados sob o currículo novo e os alunos matriculados sob o currículo antigo, exatamente como explicitado na equação 1.

\section{VARIÁVEIS DE CONTROLE}

O foco da análise é a mudança nos indicadores na medida em que o estudante está matriculado no curso de Ciências Econômicas sob o currículo de 1995.2 ou de 2009.2. No entanto, as características dos estudantes também podem influenciar nos indicadores de impacto. Para controlar ao máximo esse problema, diversas características de cada estudante foram coletadas no sistema CAGR, que formam uma matriz de covariadas.

A seguintes variáveis foram escolhidas como explicativas:

- $X_{i}$, sendo uma dummy de gênero (sexo feminino = 1 e masculino $=0$ );

- $\quad X_{i}$, sendo uma dummy se o curso foi de manhã ou à noite (noturno = 1 e matutino $=0$ );

- $\quad X_{i}$, sendo uma dummy para raça (uma para branca, uma para amarela e uma para parda);

- $\quad X_{i}$, sendo uma dummy para as formas de ingresso (vestibular $=1$ e todas as outras formas $=0)$;

- $\quad X_{i}$, sendo a idade do aluno na matrícula;

- $X_{i}$, sendo uma dummy para o estado civil do aluno (solteiro $=1$ e todos os outros estados civis $=0)$.

A tabela 1 traz um resumo das variáveis e suas estatísticas descritivas, para todas as observações do grupo de tratamento. 
Tabela 1. Estatísticas descritivas das variáveis de controle para os estudantes matriculados no currículo 2009.2

\begin{tabular}{cccccc}
\hline Variable & Obs & Mean & Std. Dev. & Min & Max \\
\hline sexo & 127 & 0,322835 & 0,469412 & 0 & 1 \\
branca & 127 & 0,889764 & 0,314424 & 0 & 1 \\
amarela & 127 & 0,007874 & 0,088736 & 0 & 1 \\
parda & 127 & 0,055118 & 0,229115 & 0 & 1 \\
idade & 127 & 21,890 & 5,2224 & 17 & 43 \\
turno & 127 & 0,543307 & 0,500094 & 0 & 1 \\
estadocivil & 127 & 0,80315 & 0,399193 & 0 & 1 \\
formadeingresso & 127 & 0,889764 & 0,314424 & 0 & 1 \\
\hline
\end{tabular}

Fonte: CAGR, elaborada pelo autor (2016)

A variável idade representa a idade média dos alunos na matrícula. Assim, a idade média na matrícula dos estudantes matriculados sob o currículo de 2009.2 é de 21,9 anos, com um desvio padrão de 5,2 anos. A variável sexo foi definida como 1 para estudantes do sexo feminino e 0 para estudantes do sexo masculino. A tabela 1 indica que, em média, há 32,2\% de estudantes do sexo feminino matriculados sob o currículo de 2009.2.

Há uma ampla maioria $(88,9 \%)$ de estudantes que entraram no curso passando pelo concurso vestibular (a variável forma de ingresso foi definida como 1 para estudantes que entraram no curso utilizando o vestibular, e 0 para todas as outras formas de ingresso).

Ao observar as estatísticas descritivas para os estudantes matriculados sob o currículo de 1995.2, é possível identificar que a idade média destes estudantes é aproximadamente 2 anos superior aos do grupo de tratamento.

Tabela 2. Estatísticas descritivas das variáveis de controle para os estudantes matriculados no currículo 1995.2

\begin{tabular}{cccccc}
\hline Variable & Obs & Mean & Std. Dev. & Min & Max \\
\hline sexo & 125 & 0,3200 & 0,4684 & 0 & 1 \\
branca & 125 & 0,8320 & 0,3754 & 0 & 1 \\
amarela & 125 & 0,0240 & 0,1537 & 0 & 1 \\
parda & 125 & 0,0800 & 0,2724 & 0 & 1 \\
idade & 125 & 23,8640 & 7,5149 & 18 & 57 \\
turno & 125 & 0,4880 & 0,5019 & 0 & 1 \\
estadocivil & 125 & 0,8480 & 0,3605 & 0 & 1 \\
formadeingresso & 125 & 0,6400 & 0,4819 & 0 & 1 \\
\hline
\end{tabular}


A média de estudantes do sexo feminino se mantém muito parecida com a média dos estudantes matriculados sob o currículo de 2009.2, 32\%. A média de estudantes solteiros se manteve parecida $(84,8 \%)$ com os estudantes matriculados sob o novo currículo.

\section{RESULTADOS}

A tabela 3 apresenta os resultados para o modelo logit com a variável dependente sendo o indicador de abandono (abandono $=1$ ).

Tabela 3. Resultados estimados para equação do modelo logit - variável dependente é Indicador de Abandono (abandono $=1)$.

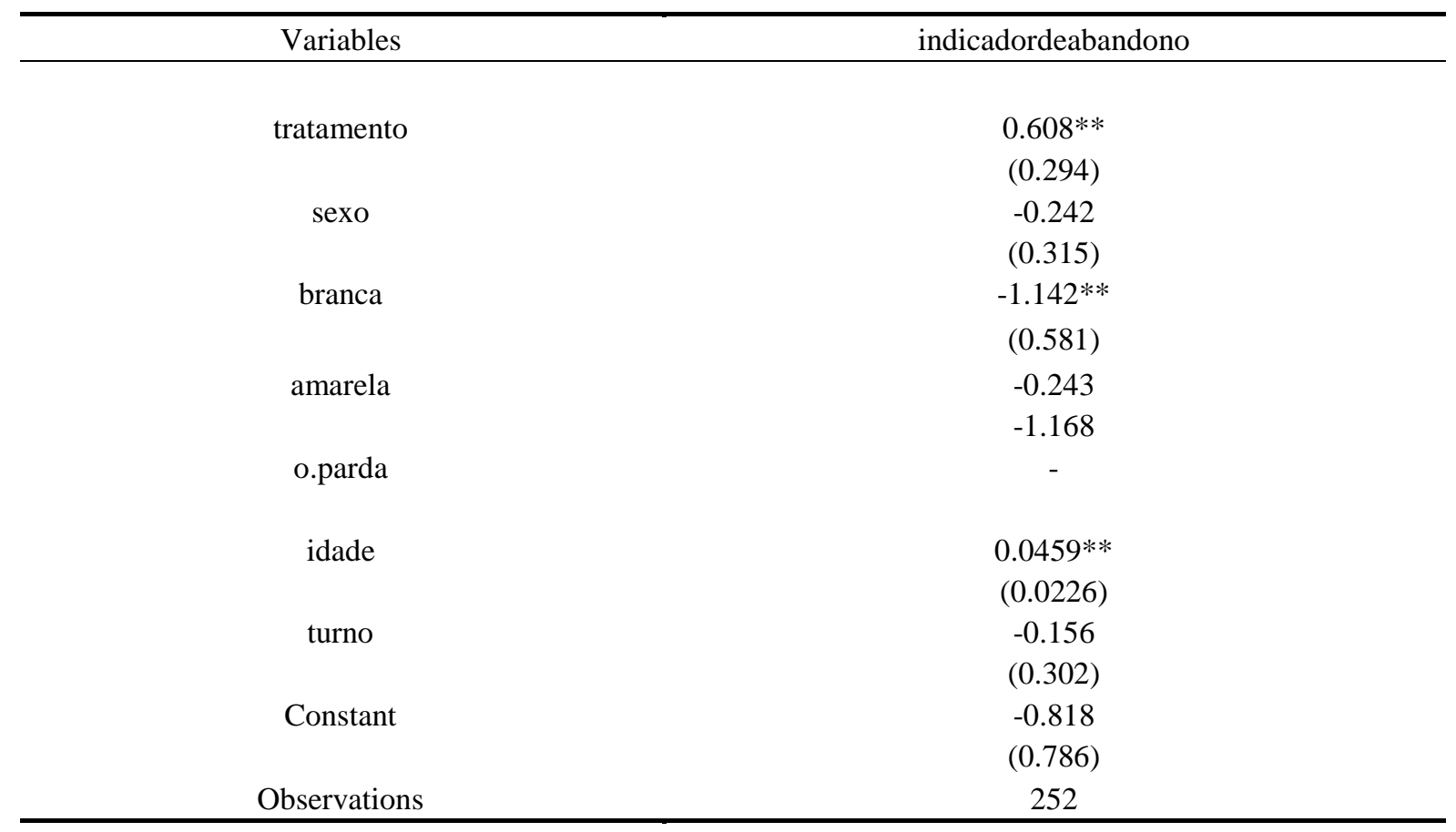

Erros padrões estão entre parênteses

As probabilidades de significância são: ${ }^{* * *} \mathrm{p}<0,01,{ }^{* *} \mathrm{p}<0,05 \mathrm{e}{ }^{*} \mathrm{p}<0,1$.

Fonte: elaborada pelo autor (2016).

Com os parâmetros estimados, é possível fazer a previsão do indicador. Como estáse interessado na média de cada grupo (de tratamento e de controle), é possível estimar a probabilidade, na média, de não abandono para cada grupo, conforme a tabela 4 abaixo. A variável parda foi omitida automaticamente por indicar multicolinearidade. 
Tabela 4. Estatísticas descritivas para a previsão utilizando a equação 4, com a variável P (previsão).

\begin{tabular}{|c|c|c|c|c|c|}
\hline Variable & Obs & Mean & Std. Dev. & Min & $\operatorname{Max}$ \\
\hline \multicolumn{6}{|c|}{ sum $\mathrm{p}$ para tratamento $=1$} \\
\hline $\mathrm{p}$ & 120 & 0,3916667 & 0,0849495 & 0,2843472 & 0,6862043 \\
\hline \multicolumn{6}{|c|}{ sum $\mathrm{p}$ para tratamento $=0$} \\
\hline $\mathrm{p}$ & 115 & 0,2956522 & 0,1002643 & 0,1778476 & 0,622717 \\
\hline
\end{tabular}

Fonte: elaborada pelo autor (2016).

Os valores estimados para a variável $P$ indicam que para os alunos que não passaram pelo tratamento, isto é, matriculados sob o currículo de 1995.2 tinham uma probabilidade de abandonar o curso, antes da quarta fase, de aproximadamente $29 \%$, enquanto que os estudantes matriculados no currículo de 2009.2 tem probabilidade de abandonar o curso antes da quarta fase de aproximadamente 39\%. Assim, a estimação indica que houve uma piora no indicador de abandono, aumentando em 10 p.p. a probabilidade de abandonar o curso após a mudança do currículo, condicionada as características observáveis de cada aluno.

Os resultados da estimação da regressão para o indicador de reprovação estão na tabela 5 .

Tabela 5. Resultados estimados para equação do modelo logit - variável dependente é Indicador de Reprovação $($ reprovação $=1)$.

\begin{tabular}{|c|c|}
\hline Variables & indicadordereprovação \\
\hline tratamento & $\begin{array}{c}1.124 * * * \\
(0.347)\end{array}$ \\
\hline sexo & $\begin{array}{c}-0.742 * * \\
(0.365)\end{array}$ \\
\hline branca & $\begin{array}{l}-0.106 \\
(0.902)\end{array}$ \\
\hline amarela & $\begin{array}{l}-0.796 \\
-1.710\end{array}$ \\
\hline parda & $\begin{array}{c}1.007 \\
-1.055\end{array}$ \\
\hline idade & $\begin{array}{l}-0.0173 \\
(0.0320)\end{array}$ \\
\hline turno & $\begin{array}{c}0.392 \\
(0.353)\end{array}$ \\
\hline Constant & $\begin{array}{c}0.376 \\
-1.170\end{array}$ \\
\hline Observations & 171 \\
\hline
\end{tabular}

Erros padrões estão entre parênteses

As probabilidades de significância são: ${ }^{* * *} \mathrm{p}<0,01,{ }^{* *} \mathrm{p}<0,05$ e ${ }^{*} \mathrm{p}<0,1$.

Fonte: elaborada pelo autor (2016). 
A partir equação da regressão, dada as características de cada estudante, permite-se chegar a previsão para o indicador de reprovação; o interesse é a média, que indicará a probabilidade de reprovação para o grupo de controle e o grupo de tratamento. A tabela 6 apresenta esses resultados.

Tabela 6. Estatísticas descritivas para a previsão utilizando a equação 4, com a variável P1 (previsão).

\begin{tabular}{cccccc}
\hline Variable & Obs & Mean & Std. Dev. & Min & Max \\
\hline & sum p para tratamento $=1$ & & & \\
p1 & 80 & 0,725 & 0,0933371 & 0,5373819 & 0,9229366 \\
& & & & & \\
sum p para tratamento $=0$ & & & & \\
& 91 & 0,494 & 0,1101798 & 0,2107641 & 0,744775 \\
\hline
\end{tabular}

Fonte: elaborada pelo autor (2016).

(tratamento - $2009.2=1$ )

A previsão do indicador de reprovação $P 1$, de acordo com as características dos estudantes, indica que, sob o currículo de 1995.2, havia uma probabilidade de reprovação de aproximadamente $49 \%$, enquanto que para os estudantes que passaram pelo tratamento, isto é, matriculados sob o currículo de 2009.2, a probabilidade de reprovação é de $72 \%$, em média.

Para o número de reprovações - uma variável de contagem - o modelo de Poisson indica a probabilidade de um estudante reprovar mais de uma vez, de acordo com suas características. Em média, se um estudante reprovar uma vez sob o currículo de 2009.2, ele tem 85\% de probabilidade de reprovar mais de uma vez, isto é, se no currículo de 1995.2 um estudante reprovava uma vez, sob o currículo novo, ele reprovaria 1,85 vezes. A tabela 7 indica os resultados da regressão de acordo com a equação 5. 
Tabela 7. Resultados estimados para equação do modelo de Poisson - variável dependente é o número de reprovações $\left(Y_{i}=0,1,2, \ldots, n\right)$

\begin{tabular}{|c|c|}
\hline Variables & indicadordereprovação \\
\hline tratamento & $\begin{array}{c}0.857 * * * \\
(0.102)\end{array}$ \\
\hline sexo & $\begin{array}{c}-0.377 * * * \\
(0.109)\end{array}$ \\
\hline branca & $\begin{array}{l}-0.127 \\
(0.274)\end{array}$ \\
\hline amarela & $\begin{array}{c}0.267 \\
(0.448)\end{array}$ \\
\hline parda & $\begin{array}{l}-0.152 \\
(0.313)\end{array}$ \\
\hline idade & $\begin{array}{l}0.000263 \\
(0.00935)\end{array}$ \\
\hline turno & $\begin{array}{c}0.364 * * * \\
(0.105)\end{array}$ \\
\hline Constant & $\begin{array}{c}0.474 \\
(0.352)\end{array}$ \\
\hline Observations & 171 \\
\hline
\end{tabular}

Erros padrões estão entre parênteses

As probabilidades de significância são: ${ }^{* *} \mathrm{p}<0,01,{ }^{* *} \mathrm{p}<0,05$ e ${ }^{*} \mathrm{p}<0,1$.

Fonte: elaborada pelo autor (2016).

Por fim, o último indicador de resultado da mudança de currículo em 2009.2 é a nota padronizada por estudante, i.e., IAA. A tabela 8 apresenta os 3 modelos de regressão linear estimados, indicando a estabilidade do $\beta 1$ (o coeficiente que ilustra o impacto da mudança do currículo no IAA).

Os três modelos são testes de diferenças de média. O modelo (1) foi estimado desconsiderando todas as características dos estudantes; ao modelo (2) foram adicionadas as variáveis de controle sexo, raça, idade, turno e estado civil, e ao modelo (3) foram adicionadas todas as variáveis de controle (as mesmas do modelo (2) mais o estado civil e a forma de ingresso). Nos 3 modelos, o $\beta 1$ (impacto da mudança do currículo) foi estatisticamente significante. O resultado da regressão indica que a mudança do currículo impactou, em média, uma diminuição de 0,46 pontos no IAA dos estudantes, condicionado as características controladas de cada aluno. 
Tabela 8. Resultados estimados para equação do modelo de Mínimos Quadrados Ordinários - variável dependente é o IAA.

\begin{tabular}{|c|c|c|c|}
\hline & (1) & (2) & (3) \\
\hline Variables & iaa & iaa & iaa \\
\hline tratamento & $\begin{array}{c}-0.383 * * \\
(0.179)\end{array}$ & $\begin{array}{c}-0.451 * * \\
(0.176)\end{array}$ & $\begin{array}{c}-0.462 * * * \\
(0.176)\end{array}$ \\
\hline sexo & & $\begin{array}{c}0.574 * * * \\
(0.190)\end{array}$ & $\begin{array}{c}0.515 * * * \\
(0.191)\end{array}$ \\
\hline branca & & $\begin{array}{c}0.182 \\
(0.478)\end{array}$ & $\begin{array}{l}0.0577 \\
(0.481)\end{array}$ \\
\hline amarela & & $\begin{array}{l}1.635^{*} \\
(0.936)\end{array}$ & $\begin{array}{c}1.478 \\
(0.934)\end{array}$ \\
\hline parda & & $\begin{array}{c}0.381 \\
(0.540)\end{array}$ & $\begin{array}{c}0.276 \\
(0.542)\end{array}$ \\
\hline idade & & $\begin{array}{c}-0.00404 \\
(0.0164)\end{array}$ & $\begin{array}{c}-0.0226 \\
(0.0191)\end{array}$ \\
\hline turno & & $\begin{array}{c}-0.409 * * \\
(0.184)\end{array}$ & $\begin{array}{c}-0.451 * * \\
(0.187)\end{array}$ \\
\hline estadocivil & & & $\begin{array}{c}-0.664 * \\
(0.343)\end{array}$ \\
\hline formadeingresso & & & $\begin{array}{c}0.141 \\
(0.207)\end{array}$ \\
\hline Constant & $\begin{array}{c}7.110 * * * \\
(0.122)\end{array}$ & $\begin{array}{c}7.036 * * * \\
(0.615)\end{array}$ & $\begin{array}{c}8.105 * * * \\
(0.872)\end{array}$ \\
\hline Observations & 171 & 171 & 171 \\
\hline R-squared & 0.026 & 0.118 & 0.139 \\
\hline
\end{tabular}

Erros padrões estão entre parênteses

As probabilidades de significância são: $* * * p<0,01, * * p<0,05$ e *p<0,1.

Fonte: elaborada pelo autor (2016).

\section{CONCLUSÕES}

Segundo o Projeto Político Pedagógico (2009), o objetivo da mudança do currículo era flexibilizar o currículo, de maneira a tornar o curso mais atraente aos estudantes; isso faria com que a evasão do curso diminuísse, e permitiria a formação de um economista mais completo. Novamente, os resultados estimados dizem respeito a comparação das duas coortes escolhidas, o que não significa que continuariam os mesmos, entre outras coortes. No entanto, caso fossem comparadas coortes mais próximas, haveria o problema da perda de graus de liberdade, e caso se escolhesse coortes mais distantes, aumentam-se os confounders.

As análises feitas neste trabalho - um exercício para avaliação na disciplina de Avaliação Econômica de Políticas Públicas - permitem concluir que, embora a probabilidade condicionada as características dos estudantes de não abandonar o curso ainda seja alta para os estudantes matriculados no currículo de 1995.2, ela aumentou 10 pontos percentuais para o 
currículo novo, variando de $29 \%$ para $39 \%$. Nesse sentido, a mudança do currículo não conseguiu atingir um de seus objetivos, para essa amostra.

No mesmo sentido, a regressão para o índice de reprovação também aumentou: passou de 49\% para estudantes matriculados no currículo de 1995.2 para $72 \%$ para estudantes do currículo de 2009.2, corroborando a hipótese de que a mudança do currículo não atingiu os seus objetivos.

Em conjunto com os dois primeiros indicadores, o número de reprovações dá ainda mais sentido às conclusões obtidas até aqui, com mudança do currículo. Há uma alta probabilidade ( $85 \%$, em média) de um estudante, condicionada as suas características, reprovar mais de uma vez sob o currículo de 2009.2 até a quarta fase. Isso pode acontecer em função da adição de matérias quantitativas na grade curricular, o que poderia tornar o curso mais difícil. Nesse sentido, um estudante matriculado no currículo de 2009.2, que já tem uma probabilidade maior de reprovar sob o currículo novo, terá uma alta probabilidade de reprovar, em média, mais de uma vez.

Por fim, a diminuição do IAA, em média, em 0,46 pontos, está em acordo com as conclusões anteriores. Esse resultado pode indicar que a mudança do currículo, embora o tenha tornado mais flexível, aumentou a dificuldade dos estudantes em obter notas mais altas. No entanto, esse indicador pode ilustrar um dos objetivos da mudança: para tornar a formação do economista mais completa, é possível que as disciplinas se tornem mais difíceis de obter notas alta, devido a ampliação do conteúdo.

No entanto, cabe frisar, há três ressalvas a se fazer, posto que podem confundir os resultados do trabalho: a entrada de novos professores, no mesmo momento da mudança do currículo, com novos métodos de ensino e de avaliação, poderia levar a uma diminuição da nota padronizada; porém, esse item não é observável, já que a dificuldade de um professor dificilmente pode ser mensurável. O segundo confounder seria o aumento da quantidade de vagas para cotistas sociais, que estudaram majoritariamente em escolas públicas, e podem ter maior dificuldade de aprendizado. No entanto, Tragtenberg et al. (2013), em seus estudos, concluíram que os estudantes que haviam ingressado na UFSC no período entre 2008.1 (quando o programa de ações afirmativas iniciou na UFSC) e 2011.2 utilizando-se de cotas para estudantes de escolas públicas tiveram números de reprovação semelhantes aos não cotistas em todos os centros da UFSC. 
A terceira e última ressalva está no fato de que não se tentou medir a efetiva flexibilização do currículo, isto é, se a proposta de flexibilizar o currículo, ofertando mais disciplinas optativas, foi efetivada.

Para o futuro, é possível melhorar este trabalho utilizando uma variável para explicitar se o estudante entrou no curso utilizando-se de cotas raciais, sociais, etc., estudando se há impacto diferente para estudantes cotistas, além de elaborar uma pesquisa com questionários para indicar se os egressos do curso, que concluíram o curso sob o currículo de 1995.2 e sob o currículo de 2009.2 estão trabalhando na área econômica (setor público ou

privado), que poderia ser um indicador de resultado da mudança do currículo. Ainda, é possível estabelecer uma medida de flexibilização do currículo, e aumentar o escopo do presente trabalho.

\section{REFERÊNCIAS}

Núcleo Docente Estruturante (NDE). $1^{\circ}$ Relatório elaborado pelo NDE do curso de Ciências Econômicas. UFSC, 2011. Disponível em:

$\langle$ http://sanson.cse.prof.ufsc.br/downloads/PrimeiroRelatorioVersaoDefinitiva2011.pdf $>$. Acesso em: 30 nov. 2016.

TRAGTENBERG, Marcelo Henrique Romano et al. Impacto das ações afirmativas na Universidade Federal de Santa Catarina (2008-2011). In: SANTOS, Jocélio Teles dos (Org.). O Impacto das Cotas nas Universidades Brasileiras: (2004-2012). Salvador: Ceao, 2013. p. 203-242.

UFSC, Coordenação do Curso de Ciências Econômicas. Projeto Político Pedagógico. 2009. Disponível em: <http://cnm.ufsc.br/files/2009/07/projeto_politico_pedagogico_02-0609.pdf>. Acesso em: 14 nov. 2016.

VIEIRA, Pedro Antonio; FÉLIX, César Augusto. O Curso de Economia da UFSC: 65 anos de história. Florianópolis: Insular, 2008. 249 p.

WOOLDRIDGE, Jeffrey M.. Introductory Econometrics: a modern approach. 5. ed. Mason: Cengage Learning, 2012. $881 \mathrm{p}$. 